\title{
Optimization of a Thermal Flow Sensor for Acoustic Particle Velocity Measurements
}

\author{
Joost W. van Honschoten, Vitaly B. Svetovoy, Gijs J. M. Krijnen, and Miko C. Elwenspoek
}

\begin{abstract}
In this paper, a thermal flow sensor consisting of two or three heated wires, the Microflown, is treated for application to acoustic measurements. It is sensitive to flow ("particle velocity"), contrary to conventional microphones that measure acoustic pressures. A numerical analysis, allowing for detailed parametric studies, is presented. The results are experimentally verified. Consequently, improved devices were fabricated, and also sensors with a new geometry consisting of three wires, instead of the usual two, of which the central wire is relatively most heated. These devices are the best performing Microflowns to date with a frequency range extending from 0 to over $5 \mathrm{kHz}$ and a minimum detectable particle velocity level of about $70 \mathrm{~nm} / \mathrm{s}$ at 2 to $5 \mathrm{kHz}$ (i.e., $3 \mathrm{~dB}$ PVL or SPL, corresponding to a pressure of $3.1 \cdot 10^{-5} \mathrm{~Pa}$ at a free field specific acoustic impedance).

[1383]
\end{abstract}

\section{INTRODUCTION}

$\mathbf{T}$ HE Microflown is an acoustic sensor based on a thermal principle [1], [2]. The sensor is mostly used for measurement purposes, and it has been applied to the measurement of one- and three-dimensional (1-, 3-D) sound intensities [3]-[6], of acoustic impedances [7], [8] and pressure [9]. The Microflown is also used as an add-on microphone for professional recording purposes [10]. The advantage of the sensor, contrary to pressure gradient microphones, is its comparatively high sensitivity to low-frequency sound waves; it can thus be used as well for the measurement of dc flows [11], and can be applied as a mass flow sensor [12]-[14].

The Microflown consists usually of two closely spaced (ca. $100 \mu \mathrm{m})$ thin wires of silicon nitride, similar to [15], with an electrically conducting platinum pattern on top of them, as shown in Fig. 1(a). The dimensions of the two wires are $1000 \times 5 \times 0.3 \mu \mathrm{m}$. The metal pattern is used as a heater and as a temperature sensor by using the temperature dependent resistance of the platinum. The wires are heated to an operational temperature between $300{ }^{\circ} \mathrm{C}$ and $400{ }^{\circ} \mathrm{C}$ by a voltage applied across the two wires in series. Due to flow across the wires, a "particle velocity," the temperature distribution around the resistors is asymmetrically altered and a temperature difference between the wires occurs, which can be measured electronically.

Recently, a good theoretical description and a model for the behavior of the device were presented [16], and an explicit analytic expression for the sensitivity was deduced [18]. However, the analytic deduction was possible by virtue of the reduction

Manuscript received July 19, 2004; revised October 1, 2004. This work was supported by the Dutch Technology Foundation STW. Subject Editor G. Benjamin Hocker.

The authors are with the Transducers Science and Technology Group, MESA+ Research Institute, University of Twente, 7500 AE Enschede, The Netherlands (e-mail: j.w.vanhonschoten@el.utwente.nl).

Digital Object Identifier 10.1109/JMEMS.2005.844848

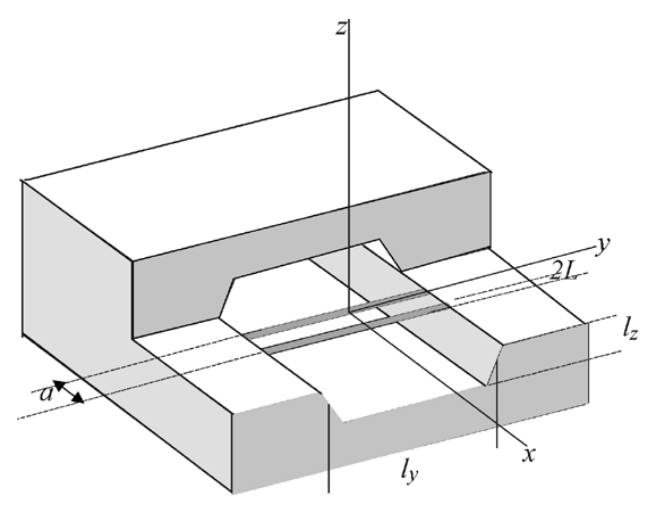

(a)

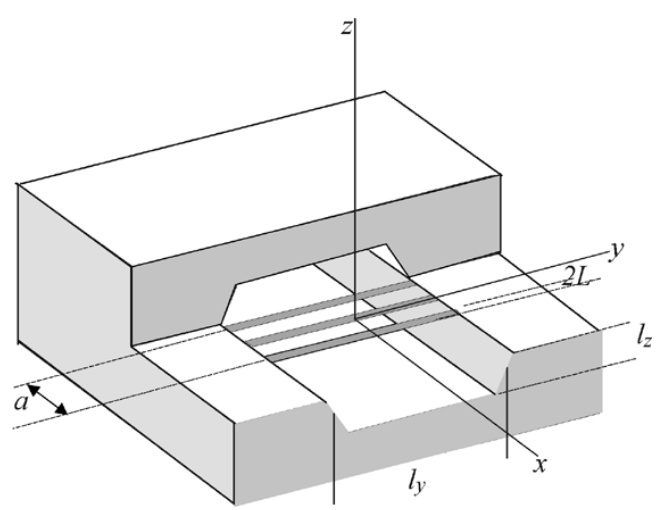

(b)

Fig. 1. (a) Model geometry of the two-wire sensor used in this paper, with cold surfaces at $z= \pm l_{z}$ above and under the heaters. (b) Model geometry of the described three-wire sensor, where the central wire acts mainly as a heater and the outer wires form the sensing wires.

of the problem to a 2-D one. Now, we release this restriction and take into account the finite length of the wires. Starting from the same basic equation, the heat diffusion equation, and following a perturbation theory approach, we find a 3-D solution for the temperature difference between the wires. This is written in the form of a double series using an expansion of orthonormal eigen functions. This representation allows for the implementation of the expression into a software program, in which the dimensions of the sensor and material parameters can easily be varied and investigated.

\section{The Temperature as a Three Dimensional Solution OF THE HEAT EQUATION}

To find the temperature distribution around the wires when there is no fluid flow, the stationary heat diffusion equation can be used. It reads

$$
-\nabla(k \nabla T)=Q
$$


where $k$ represents the thermal conductivity of the fluid, which is in principle a function of temperature $k=k(T)$, and $Q$ is the heat produced per unit of time and volume.

The geometry used is defined in Fig. 1, with the width of the heater equal to $2 L$ and $l_{y}$ the wire length. We also take into account the finite channel height of $2 l_{z}$. The surfaces at $z=$ $\pm l_{z}$ and at $y= \pm l_{y} / 2$ behave as cold walls. If the power $P$ is assumed to be generated homogeneously along the heater, $Q$ can be written as

$$
Q(x, y, z)=\frac{P}{2 L l_{y}} F(x) \delta(z) .
$$

Because of the negligible thickness of the heat source, the $z$-dependence can be represented by a $\delta$-function. First we analyze the operation with a single heater. Assume that $k$ does not depend on the temperature, which is justified if the temperature gradients are small [16], so that the heat equation becomes linear. Due to this linearity, the solution for two wires is the superposition of two displaced solutions for a single wire. A heater is oriented along the $y$-axis and its thickness in the $z$-direction is assumed to be very small. The $x$-dependence of the heat source obeys the function $F(x)$, with

$$
\begin{array}{ll}
F(x)=0 & \text { for }|x|>L ; \\
F(x)=1 & \text { for }|x|<L .
\end{array}
$$

With the assumption that the heat flux through the wire ends to the channel walls is small compared to the total dissipated power in the heater, the heat equation becomes

$$
\left(\partial_{x}^{2}+\partial_{y}^{2}+\partial_{z}^{2}\right) T=-\frac{P}{2 l_{y} k L} F(x) \delta(z) .
$$

To simplify this equation, the function $F(x)$ is changed into a $\delta$-function as well: if the width $2 L$ is not too large, $F(x) / 2 L$ can be replaced by $\delta(x)$. Introducing the normalized variables $\xi=x / l_{z} ; \eta=2 y / l_{y} ; \zeta=z / l_{z}$, where $l_{z}$ is half of the channel height, changes the equation into

$$
\left(\partial_{\xi}^{2}+\left(\frac{2 l_{z}}{l_{y}}\right)^{2} \partial_{\eta}^{2}+\partial_{\zeta}^{2}\right) T=-\frac{P l_{z}^{2}}{l_{y} k} \delta(x) \delta(z)=-\frac{P}{l_{y} k} \delta(\xi) \delta(\zeta) .
$$

Taking into account that the solution should obey the boundary conditions that the temperature is zero at the channel surfaces $z= \pm l_{z}$ and $y= \pm l_{y} / 2$, we write $T(\xi, \eta, \zeta)$ as an expansion in orthonormal harmonic eigenfunctions in $\eta$ and $\zeta$

$$
\begin{aligned}
T(\xi, \eta, \zeta) & =\sum_{n m=0}^{\infty} T_{n m}(\xi) \cos \left(\lambda_{n} \zeta\right) \cos \left(\lambda_{m} \eta\right), \\
\lambda_{i} & =\frac{\pi}{2}(2 i+1) \quad i=n, m .
\end{aligned}
$$

Substituting this expansion into (5), and taking into account the boundary conditions and the matching condition at $\zeta=0$, gives for $T_{n m}(\xi)$

$$
\begin{aligned}
T_{n m}(\xi) & =\frac{T_{0}(-1)^{m}}{\lambda_{m} \sigma_{n m}} e^{-\sigma_{n m}|\xi|} \\
T_{0} & =\frac{P}{k l_{y}}, \quad \sigma_{n m}=\sqrt{\lambda_{n}^{2}+\left(\frac{2 l_{z}}{l_{y}}\right)^{2} \lambda_{m}^{2}} .
\end{aligned}
$$

\section{The Temperature DifFERENCE DuE TO THE FluID VELOCITY}

The output signal of the operating Microflown, when it is exposed to acoustic waves or fluid flows, is a voltage. This output voltage results from a resistance difference between the two wires that is caused by the temperature difference between the wires. For small temperature variations, the resistance change of a wire is in good approximation linearly proportional to its temperature change: if the resistance at a certain temperature $T_{0}$ is defined as $R_{0}$, then the resistance at a temperature $T, R(T)$, is in first order

$$
R(T)=R_{0}\left(1+\alpha\left(T-T_{0}\right)\right)
$$

in which $\alpha$ represents the resistance temperature coefficient in $\mathrm{K}^{-1}$. The differential output voltage $u_{0}$, which is usually amplified and then measured, is directly related to the resistance variation $\Delta R$ between the two wires. The voltage $U_{0}$ is applied over both wires in series so that the voltage drop over one heater is $U_{0} / 2$. The output voltage $u_{0}$ is therefore proportional to the temperature difference $\Delta T$ between the wires [1], [16]

$$
u_{0}=\frac{U_{0}}{2} \alpha \Delta T
$$

So, we now have to find an expression for $\Delta T$. In order to calculate this temperature difference due to the presence of a small term concerning the velocity $\vec{v}$, we can consider the convection term as a perturbation to the stationary temperature profile of the wire. In the case of a moving gas in the channel, the heat equation for the temperature becomes

$$
\rho c_{p}\left(\partial_{t} T+\vec{v} \cdot \nabla T\right)-\nabla(k \nabla T)=Q
$$

with the vector $\vec{v}$ the gas (or fluid) velocity, $c_{p}$ the heat capacity of the gas at constant pressure, and the heat power density $Q$ defined as before (25). ${ }^{1}$ When the temperature gradients are not too high so that the inhomogeneities of the heat conduction coefficient $k$ can be neglected again, and when the gas velocities are relatively small, the solution of (10) consists of the temperature from (7), plus a small correction $\delta T$ proportional to $\vec{v}$. This correction, or perturbation term, $\delta T$, obeys the nonhomogeneous equation

$$
\partial_{t} \delta T-D \nabla^{2} \delta T=-v \partial_{x} T
$$

with $D=k / \rho c_{p}$, the thermal heat diffusion coefficient. Since the velocity is directed in the $x$-direction along the channel, $\vec{v}=(v, 0,0)$ and the term $\vec{v} \cdot \nabla T$ from (10) appears as $v \partial_{x} T$. The amplitude $v$ of the particle velocity (the instantaneous flow) of the acoustic wave depends on fluid parameters such as the density and is thus temperature dependent, so that it cannot be considered to be constant along the channel. For a harmonically varying wave in the channel with a velocity $v$ corresponding to

\footnotetext{
${ }^{1}$ The effect of free convection is small in our case. Free convection is important in situations where the Grashof number $G r$ is larger than 1000 [29]. For the current dimensions and temperatures, $G r \ll 1$ and one can neglect its influence [17].
} 
an acoustic pressure $\delta p_{0} e^{i \omega t}$ at room temperature $T_{r}$, one can write in good approximation

$$
v(\xi, \eta, \zeta, t)=v_{0} \frac{T(\xi, \eta, \zeta)}{T_{r}} e^{i \omega t} \quad v_{0}=\frac{\delta p_{0}}{\rho\left(T_{r}\right) c}
$$

with $\rho\left(T_{r}\right) c$ the acoustic impedance $z$ at room temperature $T_{r}$ $\left(z=448 \mathrm{~kg} / \mathrm{m}^{2} \mathrm{~s}\right.$ at $\left.T_{r}=293 \mathrm{~K}\right)$. For the latter relation between velocity and pressure, the "free field" condition was assumed to be fulfilled. Since the expression for $v$ has to obey the boundary conditions and vanish on the channel walls, ${ }^{2}$ it is written as an expansion in harmonics in $\zeta$ and $\eta$

$$
v(\xi, \eta, \zeta, t)=e^{i \omega t} \sum_{n, m}^{\infty} v_{n m}(\xi) \cos \left(\lambda_{m} \eta\right) \cos \left(\lambda_{n} \zeta\right)
$$

of which the amplitudes $v_{n m}(\xi)$ still have to be determined. The Reynold's numbers for the system are typically very low $\left(\operatorname{Re} \leq 10^{-3}\right)$, so that for a rectangular channel and a fully developed flow the velocity distribution is parabolic in $y$ - and $z$-directions [19] and can be approximated by only the first harmonics. Therefore, an adequate expression for the velocity becomes [16]

$$
v(\xi, \eta, \zeta, t)=v_{0} \frac{T(\xi, \eta, \zeta)}{T_{r}} e^{i \omega t} \cos \left(\frac{\pi}{2} \eta\right) \cos \left(\frac{\pi}{2} \zeta\right)
$$

which clearly expresses the position and temperature dependence of the particle velocity within the sensor. The stationary temperature distribution $(v=0)$ is found from the heat equation, (5). For simplification, the calculations are performed first for the situation of only one wire at $\xi=0$. Subsequently, the solutions for $T$ and for $\delta T$ can be obtained with parametric shifts to $\xi=+\xi_{1}$ and $\xi=-\xi_{1}$. (For a sensor with wire separation $a$, $\xi_{1}=a / 2 l_{z}$.) For the temperature distribution, one can write as before the expansion in harmonics from (6). For harmonic oscillations in time of the form $\delta T=\delta T(\xi, \eta, \zeta) e^{i \omega t}$, the equation for $\delta T$ becomes

$$
i \omega \delta T-D \nabla^{2} \delta T=-v \partial_{x} T
$$

Scaling to dimensionless variables changes this into

$$
\left(\partial_{\xi}^{2}+\left(\frac{2 l_{z}}{l_{y}}\right)^{2} \partial_{\eta}^{2}+\partial_{\zeta}^{2}\right) \delta T-i \omega \frac{l_{z}^{2}}{D} \delta T=-\frac{v}{\bar{v}} \partial_{\xi} T, \text { with } \bar{v} \equiv \frac{D}{l_{z}} .
$$

The thermal diffusion constant $D$ depends on the temperature via the fluid density $\rho$. It also depends on the temperature via $k(T)$ and $c_{p}(T)$, but these temperature dependences are small compared to the temperature dependence of $\rho$. The fluid velocity is not constant within the sensor either, since it is temperature dependent also. Nevertheless, in the ratio $v / \bar{v}$ the temperature dependence disappears so that one can take as a good approximation for the velocity $v_{0} e^{i \omega t} \cos ((\pi / 2) \eta) \cos ((\pi / 2) \zeta)$, and use $D$ at room temperature.

\footnotetext{
${ }^{2}$ The assumption that on the channel walls $\eta= \pm 1, \zeta= \pm 1, v=0$, is called the "no-slip condition" [19], [20].
}

For the correction term $\Delta T$, a double expansion similar to that for $T$ can be made. It is

$$
\begin{aligned}
\delta T(\xi, \zeta, \eta) & =\sum_{n, m=0}^{\infty} \delta T_{n m}(\xi) \cos \left(\lambda_{n} \zeta\right) \cos \left(\lambda_{m} \eta\right) \\
\lambda_{i} & =\frac{\pi}{2}(2 i+1) \quad i=n, m .
\end{aligned}
$$

Using the expansion for $\delta T(\xi),(17)$, then one finds, with the notation $\delta T^{\prime \prime} \equiv \partial^{2} T / \partial \xi^{2}$

$$
\delta T_{n m}^{\prime \prime}-\sigma_{n m}^{2} \delta T_{n m}-i \frac{\omega l_{z}^{2}}{D} \delta T_{n m}=\frac{v_{0}}{\bar{v}} \partial_{\xi} T_{n m}(\xi) .
$$

To be fully correct, one should take for $v_{0}$ in (18) the full expansion in harmonics, $\sum_{i, j=0}^{\infty} v_{i j} \cos \left(\lambda_{i} \eta\right) \cos \left(\lambda_{j} \zeta\right)$, or at least for a parabolic flow profile, the first coefficient of this series, as in (14). For each $\delta T_{n m}$, the velocity term at the right-hand side (RHS) of (18) will thus contain a summation over $i$ and $j$. In [16] this total summation was evaluated explicitly, and it was concluded that if the full expansion of $v$ is replaced by the single value $v_{0}$ the result changes less than $1 \%$. Therefore, we apply this simplification and set $v(\xi, \eta, \zeta)=v_{0}$. Since (7) gives $T_{n m}(\xi)=\left(T_{0}(-1)^{m} / \lambda_{m} \sigma_{n m}\right) e^{-\sigma_{n m}|\xi|}, T_{0}=P / k l_{y},(18)$ can be rewritten so that one obtains for $\delta T_{n m}(\xi)$

$$
\begin{aligned}
\delta T_{n m}^{\prime \prime}-K_{n m}^{2} \delta T_{n m} & =-\operatorname{sign}(\xi) \frac{v_{0}}{\bar{v}} \frac{(-1)^{m}}{\lambda_{m}} T_{0} e^{-\sigma_{n m}|\xi|} \\
K_{n m}^{2} & =\sigma_{n m}^{2}+i \bar{f}
\end{aligned}
$$

with $\bar{f} \equiv \omega l_{z}^{2} / D$ a normalized frequency. The total solution of this nonhomogeneous equation is the sum of the solution of the homogeneous equation and a particular solution of the nonhomogeneous one. Taking into account the boundary conditions for $\delta T(\xi)$ and $\delta T^{\prime}(\xi)$ one then finds for $\delta T_{n m}$

$\delta T_{n m}(\xi)=\operatorname{sign}(\xi) \frac{1}{i \bar{f}} \frac{v_{0}}{\bar{v}} \frac{(-1)^{m}}{\lambda_{m}} T_{0}\left(e^{-\sigma_{n m}|\xi|}-e^{-K_{n m}|\xi|}\right)$.

If there are now two wires, symmetrically positioned at $\xi=$ $-\xi_{1}$ and $\xi=+\xi_{1}$, this becomes

$$
\begin{aligned}
\delta T_{n m}(\xi)= & \operatorname{sign}\left(\xi-\xi_{1}\right) \frac{1}{i \bar{f}} \frac{v_{0}}{\bar{v}} \frac{(-1)^{m}}{\lambda_{m}} \\
& \times T_{0}\left(e^{-\sigma_{n m}\left|\xi-\xi_{1}\right|}-e^{-K_{n m}\left|\xi-\xi_{1}\right|}\right) \\
& +\operatorname{sign}\left(\xi+\xi_{1}\right) \frac{1}{i \bar{f}} \frac{v_{0}}{\bar{v}} \frac{(-1)^{m}}{\lambda_{m}} \\
& \times T_{0}\left(e^{-\sigma_{n m}\left|\xi+\xi_{1}\right|}-e^{-K_{n m}\left|\xi+\xi_{1}\right|}\right) .
\end{aligned}
$$

The temperature difference between the wires at $\xi=-\xi_{1}$ and $\xi=\xi_{1}$ is, therefore, given by

$$
\begin{aligned}
\Delta T\left(v_{0}\right) & =\delta T\left(\xi_{1}\right)-\delta T\left(-\xi_{1}\right) \\
& =\sum_{n, m=0}^{\infty} \frac{2}{i \bar{f}} \frac{v_{0}}{\bar{v}} \frac{1}{\lambda_{m}^{2}} T_{0}\left(e^{-2 \sigma_{n m} \xi_{1}}-e^{-2 K_{n m} \xi_{1}}\right) .
\end{aligned}
$$




\section{The Influence of the HeAt CAPACity OF THE WiRES}

When the heat capacities of the two wires cannot be neglected, an additional term will appear in the heat diffusion equation. Equation (11) becomes then

$$
\begin{aligned}
\partial_{t} \delta T-D \nabla^{2} \delta T= & -v \partial_{x} T+H(z) \frac{\left(\rho c_{p}\right)_{\text {sensor }}}{\left(\rho c_{p}\right)_{\text {air }}} \\
& \times\left(F(x-a) \partial_{t} \delta T+F(x+a) \partial_{t} \delta T\right)
\end{aligned}
$$

with

$$
\begin{array}{ll}
H(z)=0 & \text { for }|z|>\frac{h}{2} \\
H(z)=1 & \text { for }|z|<\frac{h}{2}
\end{array}
$$

in which $h$ represents the thickness of the wires. For small $h$, the step function $H(z)$ can be replaced by $h \delta(z)$. With the introduction of $\beta=\left(h / l_{z}\right)\left(\left(\rho c_{p}\right)_{\text {sensor }} /\left(\rho c_{p}\right)_{\text {air }}\right)$, it can be deduced as shown in (25) at the bottom of the page.

In obtaining this expression, the finite width $2 \xi_{0}$ of the heater was taken into account by averaging over the heater width, so that the sum in the denumerator becomes convergent.

\section{THE THREE-Wire CONFIGURATION}

As we will see later, a configuration of the Microflown that consists of three instead of two wires is often favorable to a twowire sensor. Its working principle is based on a separation of the heating and sensing functionality. The sensor consists of three wires, of which the central wire acts as a heater and the two outer wires provide the sensing function. An example of a three-wire sensor is seen in Fig. 2. Power is predominantly dissipated in the central wire, while the sensing wires have a relatively low temperature and thus a relatively low noise level. Moreover, the heating wire can be designed such that much power in it can be dissipated and that its heat capacity is of minor importance. This concept is simply implemented in the present model.

Assume three wires of equal normalized width $2 \xi_{0}$ at $-\xi_{1}, 0$, and $\xi_{1}$. The model geometry is shown in Fig. 1(b). The fraction of the total dissipated power in the central wire is $r_{1}$, while each outer wire dissipates a fraction $r_{2}$, so $r_{1}+2 r_{2}=1$. The numerator in expression (25) then changes into

$$
\begin{aligned}
\sum_{n=0}^{\infty} r_{1} & \left(\left(\frac{\sinh \sigma_{n m} \xi_{0}}{\sigma_{n m} \xi_{0}}\right)^{2} e^{-\sigma_{n m} \xi_{1}}-\left(\frac{\sinh K_{n m} \xi_{0}}{K_{n m} \xi_{0}}\right)^{2} e^{-K_{n m} \xi_{1}}\right) \\
+r_{2} & \left(\left(\frac{\sinh \sigma_{n m} \xi_{0}}{\sigma_{n m} \xi_{0}}\right)^{2} e^{-2 \sigma_{n m} \xi_{1}}\right. \\
& \left.-\left(\frac{\sinh K_{n m} \xi_{0}}{K_{n m} \xi_{0}}\right)^{2} e^{-2 K_{n m} \xi_{1}}\right) .
\end{aligned}
$$

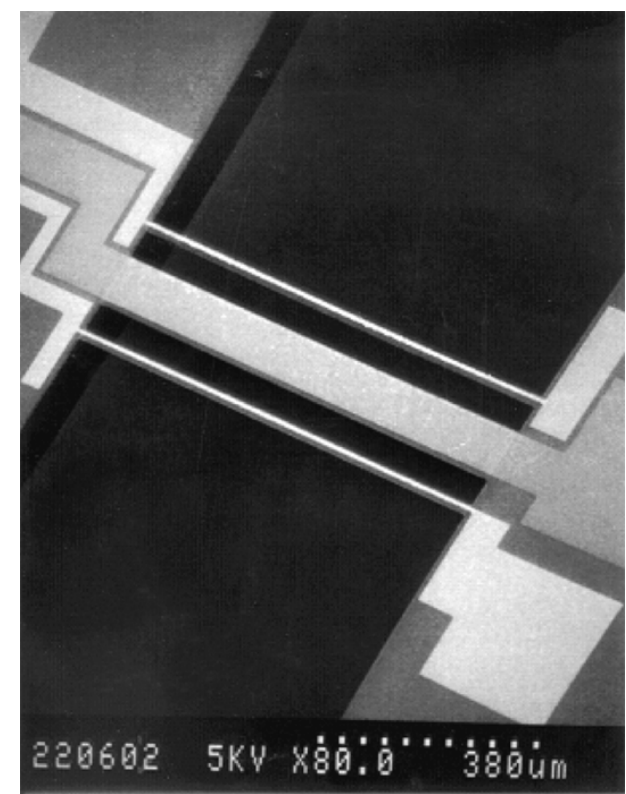

Fig. 2. SEM photograph of an example of a three-wire microflown, with one thick central wire (the heater) and two much thinner sensing wires.

For $r_{1}=0$ and $r_{2}=1 / 2$, one obtains again the expression for the two-wire sensor.

In a numerical and experimental investigation of the threewire concept and the influence of the relative powers on the sensitivity, these ratios $r_{1}$ and $r_{2}$ can easily be varied.

\section{EXPERIMENTAL ANALYSIS OF THE OUTPUT SignAL}

In acoustic calibration measurements, the output voltage, (9), is measured as a function of frequency. The function for $\Delta T$ can be characterized by three properties when it is plotted double logarithmically: the low-frequency absolute value, i.e., the intersection with the sensitivity axis, a first characteristic frequency $f_{D}$ determining where the function falls off, and a second corner frequency due to the heat capacity of the wires [16], [17]. All three parameters should be as high as possible. So, our purpose is to optimize expression (25). In this paper, we concentrate on the low-frequency sensitivity and the first characteristic frequency. The dependence of the second corner frequency on the thermal mass (it is inversely proportional to the heat capacity) is obvious, and was experimentally verified in [17], [18]. By making the wires as thin as possible, the corner frequency should become high. Here it was kept constant in all investigations, and equal to $3.0 \mathrm{kHz}$.

The output signal $u_{0}$ can be written as a function of mutually independent variables. Since many independent parameters like

$$
\begin{aligned}
\Delta T & =\delta T\left(\xi_{1}\right)-\delta T\left(-\xi_{1}\right) \\
& =\frac{1}{i \bar{f}} \frac{2 v_{0}}{\frac{D}{l_{z}}} T_{0} \sum_{m=0}^{\infty} \frac{1}{\lambda_{m}^{2}} \frac{\sum_{n=0}^{\infty}\left(\left(\frac{\sinh \sigma_{n m} \xi_{0}}{\sigma_{n m} \xi_{0}}\right)^{2} e^{-2 \sigma_{n m} \xi_{1}}-\left(\frac{\sinh K_{n m} \xi_{0}}{K_{n m} \xi_{0}}\right)^{2} e^{-2 K_{n m} \xi_{1}}\right)}{1+i \beta \bar{f} \sum_{n=0}^{\infty} \frac{1}{K_{n m}^{2}}\left(\frac{\sinh K_{n m} \xi_{0}}{K_{n m} \xi_{0}}\left(e^{-K_{n m} \xi_{0}}+e^{-2 K_{n m} \xi_{1}} \sinh \left(K_{n m} \xi_{0}\right)\right)-1\right) .}
\end{aligned}
$$






Fig. 3. Numerically calculated sensitivity according to the model for varying mutual wire distance $a$. The etch depth is $l_{z}=200 \mu \mathrm{m}$; wire length $l_{y}=$ $500 \mu \mathrm{m}, P=45 \mathrm{~mW}$ and $R_{0}=2.0 \mathrm{k} \Omega$.

$\alpha$ and $R_{0}$ can be considered as constants on which the dependence of $u_{0}$ is trivial, we analyze the behavior of $u_{0}$ as a function of the following dimensionless parameters: the normalized frequency $f /\left(D / 2 \pi l_{z}^{2}\right)$ and the geometrical variables $a / l_{z}$ and $l_{z} / l_{y}$. Additionally, the ratio $P / l_{y}$, the power per unit length, is an important scale factor. Apart from the optimization of the (low-frequency) output signal $u_{0}$, it is important to consider the "sensitivity-to-noise" (S/N) ratio of the output. This ratio, generally referred to as the "signal-to-noise" ratio (SNR), is the actual quantity to be maximized. The main contribution to the noise is the resistance or Johnson noise [21], [22]: for a resistor $R$ at absolute temperature $T$ the voltage fluctuations in a frequency interval $\Delta f$ are: $\left\langle u_{\text {noise }}^{2}\right\rangle_{(f, f+\Delta f)}=(4 k T R) \Delta f$, with $k$ the Boltzmann constant. Since the wire resistance of the sensor is also temperature dependent (8), the noise increases with the wire temperature.

To compare the theoretically and the numerically obtained results to the sensitivity curve of the sensor for different geometries, several devices with varying etch depths, mutual wire distances and wire lengths were designed. We fabricated two-wire sensors with $l_{z}=40,80,120,240$, and $300 \mu \mathrm{m}, l_{y}=500$ and $1000 \mu \mathrm{m}$, and $a=50,80,100,200$, and $300 \mu \mathrm{m}$. The sensors were characterized in a standing wave tube with a reference microphone in it, as described in [1], [8]. We thus obtained the required sensitivity curves $S(f)$ in $\mathrm{V} / \mathrm{Pa}$ or in $\mathrm{Vs} / \mathrm{m}$. Using a mathematic software program, we wrote a script that calculates explicitly $u_{0}$ with $\Delta T$ from (25), where the various parameters (such as $l_{y}, a, l_{z}, P$ and $R$ ) were taken as input variables. Considering the relevant mutually independent variables in this expression, we varied subsequently $a / l_{z}, P / l_{y}$ and $l_{z} / l_{y}$.

Keeping the power per unit length, $P / l_{y}$ constant, the sensitivity as a function of frequency was calculated. Fig. 3 shows some representative functions for different parameters.

\section{A. The Low-Frequency Sensitivity}

For given etch depth $l_{\mathrm{z}}$, it is seen in Fig. 3 that there is an optimum value for $a$, for which the low-frequency sensitivity $S_{f=0}$ is maximal. Additionally, the value of $a$ determines the first characteristic frequency. For large $l_{z}$ where the wires can be approximated to be in free space, this characteristic frequency

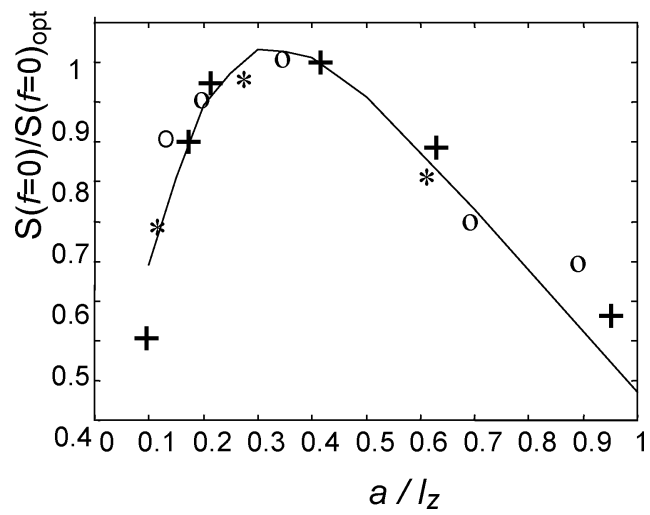

Fig. 4. Numerically calculated low-frequency sensitivity $S(f=0)$ as a function of the ratio $a / l_{z}$ (dotted line). The crosses denote experimental results from device geometries with etch depths $l_{z}=240 \mu \mathrm{m}(+), l_{z}=300 \mu \mathrm{m}$ (*), and $l_{z}=500 \mu \mathrm{m}$ (o). The vertical scale has been normalized to the optimum value of $S(f=0)$.

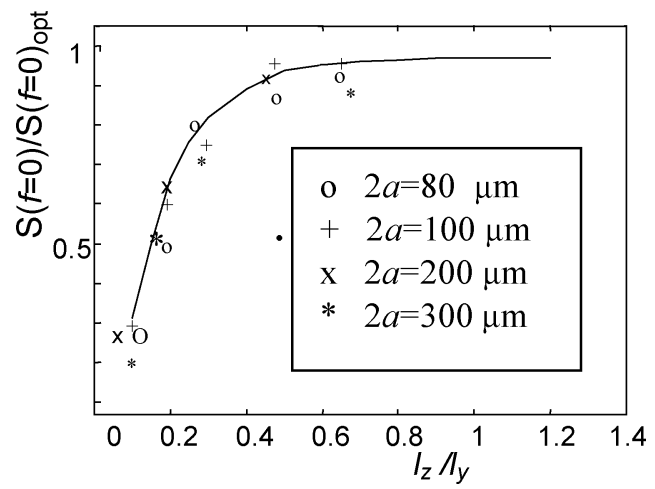

Fig. 5. Numerically calculated sensitivity $S(f=0)$ as a function of the ratio $l_{z} / l_{y}$ (dotted line). The marks indicate experimentally obtained results from devices with $l_{y}=500 \mu \mathrm{m}, l_{z}=40,80,120,240$, or $300 \mu \mathrm{m}$, and $a=80$, 100,200 or $300 \mu \mathrm{m}$. The vertical scale has been normalized to the value of $S(f=0)$ at $l_{z}=l_{y}$.

equals $f_{D}=D / 2 \pi a^{2}$ [16]-[18]. To find the value of the ratio $a / l_{\mathrm{z}}$ for which $S_{f=0}$ is maximum, we optimized the expression for $u_{0}$ for $a / l_{\mathrm{z}}$. It can be seen that this value is independent of $l_{y}$, and the optimal ratio was calculated to be approximately $\left(a / l_{z}\right)_{\text {opt }} \approx 0.3$ (Fig. 4). From the figure it is concluded that for given $l_{z}$, an optimum value for $a$ exists. However, the calculations also show that for given $a$, the sensitivity $S_{f=0}$ monotonously increases with $l_{z}$ up to $l_{z}=l_{y}$. This influence of the ratio $l_{z} / l_{y}$ on the low frequency sensitivity was investigated and is shown in Fig. 5. For ratios $l_{z} / l_{y}$ above approximately 0.6 , the low-frequency sensitivity does not increase significantly.

\section{B. Frequency Dependent Sensitivity}

As aforementioned, the mutual wire distance $a$ is of direct influence on the characteristic frequency of the sensitivity curve $S(f)$. A decrease of $a$ increases the bandwidth. The result of a series of measurements on a number of sensors with $l_{z}=$ $240 \mu \mathrm{m}$ and varying $a$, is shown in Fig. 6 . The experimentally determined sensitivity curves were approximated by a second order low pass frequency response. The lowest corner frequency $f_{c}$ of this function is proportional to the thermal diffusion frequency $f_{D}=D / 2 \pi a^{2}$. It is plotted as a function of $a$ in Fig. 6 on a double logarithmic scale. For wires in free space, the analytic model [18] predicts the relationship $f_{c} \propto a^{-2}$, which 




Fig. 6. Characteristic frequency $f_{c}$ as a function of the distance between the wires. The acoustically obtained sensitivities were fitted by a second order frequency response with lowest corner frequency $f_{c}$. The axes are plotted logarithmically; the line shows the theoretical dependence $f_{c} \propto a^{-2}$.

is represented by the line. The experimental points have rather large error bars, due to the fact that the fit functions are good approximations. The line represents the analytic model for wires in free space, while the experimental results refer to devices with a channel surface below acting as heat sink. Nevertheless, the behavior of $f_{c} \propto a^{-2}$ appears for these sensors. The influence of the channel bottom on $f_{D}$ appears to be small.

From the results of Figs. 4, 5 and 6, it is concluded that $S_{f=0}$ has an optimum for a ratio of $a$ and $l_{z}$ as $a \approx 0.3 l_{z}$. Since the signal also increases with $l_{z}$ up to $l_{y}=l_{z}$, the etch depth $l_{z}$ should be chosen as $l_{z} / l_{y} \approx 0.5$ to 1 . A certain required characteristic frequency thus determines the choice of $a$, from which $l_{z}\left(a \approx 0.3 l_{z}\right)$ and then $l_{y}\left(l_{y}<2 l_{z}\right)$ are chosen.

\section{The Three-Wire CONFIGURATION}

\section{A. The Concept}

As aforementioned, the most relevant quantity to maximize is the sensitivity to noise ratio, the quotient $\mathrm{S} / \mathrm{N}$, with $N$ the noise voltage $N=\sqrt{\left\langle u_{\text {noise }}^{2}\right\rangle}$ in the sensing wires. As a first approximation for this noise voltage, it is assumed that the noise of the heated wires is mainly determined by the Johnson noise level associated with the wire resistance $R$. Other sources of noise, in particular low frequency noise with $1 / f$ shaped spectral densities $(1 / f$ or flicker noise) that are ubiquitous in many systems like thin metal films, metaloxide layers, wires, and resistors, and about which much research has been done [e.g., [22]-[28]], are initially not taken into account. Since the resistance noise is temperature dependent and depends on $R$, which is itself a function of temperature, an increasing power in a wire leads to an increasing noise voltage. With respect to the noise, a relatively low temperature in the sensing wires is therefore favorable. The signal, however, increases with the total dissipated power.

The introduced new configuration for the Microflown, the three-wire sensor, was experimentally analyzed by considering $S_{f=0}$ as a function of the relative heater power $r_{1}$. The dashed curve in Fig. 7 shows an optimum at approximately $r_{1}=0.8$. With respect to the SNR, defined as the low-frequency sensitivity divided by the noise level due to Nyquist noise, an even

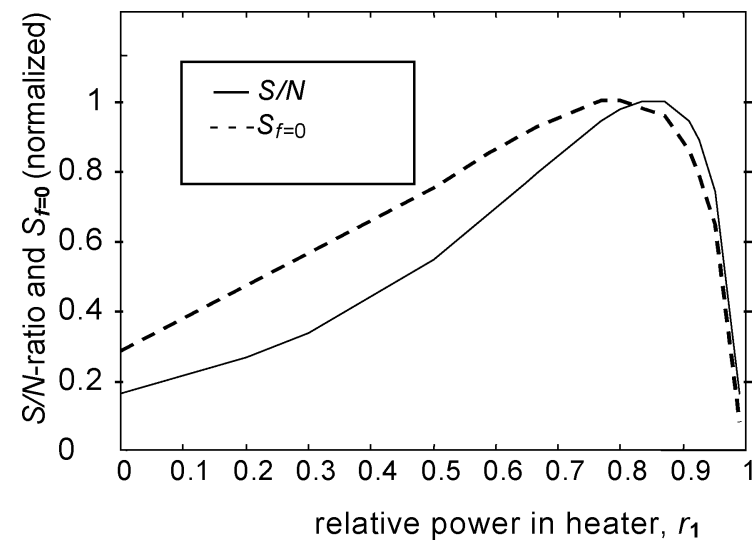

Fig. 7. Relative low-frequency sensitivity $S_{f=0}$ as a function of relative heater power $r_{1}$ (dotted line) and the SNR $S_{f=0} / N$, with $N$ the Nyquist noise level (drawn line). Both $\mathrm{S} / \mathrm{N}$ and $S_{f=0}$ have been normalized to their value at $r_{1}=0$, i.e., the two-wire case.

higher ratio $r_{1}$ is favorable. The drawn line in Fig. 7 shows $S_{f=0} / N$ as a function of $r_{1}$ and it is seen to have its optimum at $r \approx 0.85$.

For a matter of fact, in the three-wire configuration with a heater-sensing wire separation of $a / 2$ the characteristic frequency is mainly determined by the distance $a / 2$, while for the corresponding two-wire device this frequency is determined by the separation $a$. The frequency bandwidth thus increases by placing a heater between the sensing wires. The frequency dependence of a three-wire device of separation $a$ between the sensing wires greatly corresponds to that of a two-wire device with wire separation $a / 2$. However, the optimization strategy with respect to $a, l_{z}$ and $l_{y}$ remains the same as for two-wire sensors.

\section{B. Experimental Characterization of the Three-Wire Device}

Since the output signal increases with the power per unit length [(7) and (25)], a high power dissipation in the wires is favorable. However, the wire cannot stand temperatures that are too high. Usually the temperature of the sensing wire, designed to be thin, should not increase above approximately $650 \mathrm{~K}$. It is found experimentally that the maximum power per unit wire length is about $30 \mathrm{~W} / \mathrm{m}$. An advantage of the three-wire configuration is that the heater can be designed to be thicker, so that it can stand a higher temperature, currently about $800 \mathrm{~K}$. Moreover a high thermal heat capacity reduces the noise from thermal and resistance fluctuations.

Three three-wire devices were designed with $l_{y}=1.0 \mathrm{~mm}$, $l_{z}=200 \mu \mathrm{m}$, and $a=100,200$, and $300 \mu \mathrm{m}$. The wire resistances were $R_{\mathrm{s} 1}=R_{\mathrm{s} 2}=3.47 \mathrm{k} \Omega$ and $R_{\mathrm{H}}=260 \Omega$. The heater voltage was adjusted so that the heater power $P_{h}$ gradually increased from 0 to $58 \mathrm{~mW}$. For each value of $P_{h}$, the power in the sensors $P_{s}$ was varied from 2 to $21 \mathrm{~mW}$. The measurement results are summarized in Figs. 8 and 9. These points are compared to the theoretical curves. It can be concluded that the use of the three wire configuration largely improves the low-frequency sensitivity at constant total power. Fig. 8 illustrates that the optimum power ratio $r \approx 0.85$ yields an approximately two times higher sensitivity $S_{f=0}$ than the ratio $r=0$, which actually represents the two-wire device. 


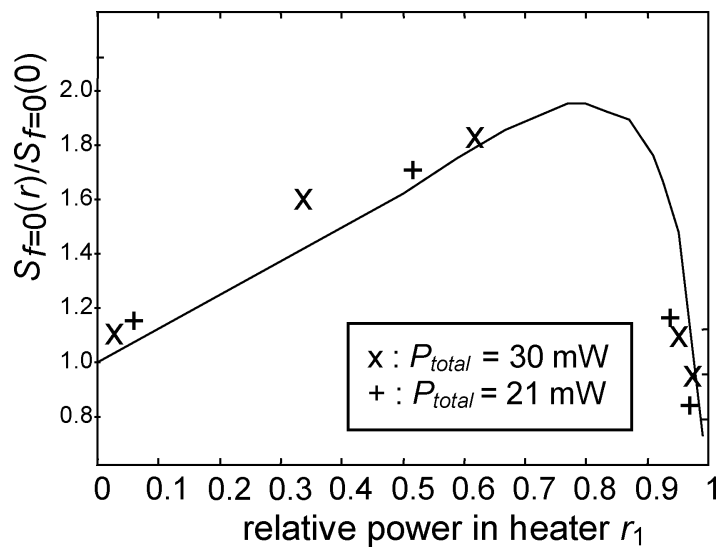

Fig. 8. Relative low-frequency sensitivity $S(f=0)$ as a function of relative heater power $r_{1}$, (line) according to model calculations, and the experimentally obtained points for $P_{\text {total }}=P_{h}+2 P_{s}=21$ and $30 \mathrm{~mW}$.

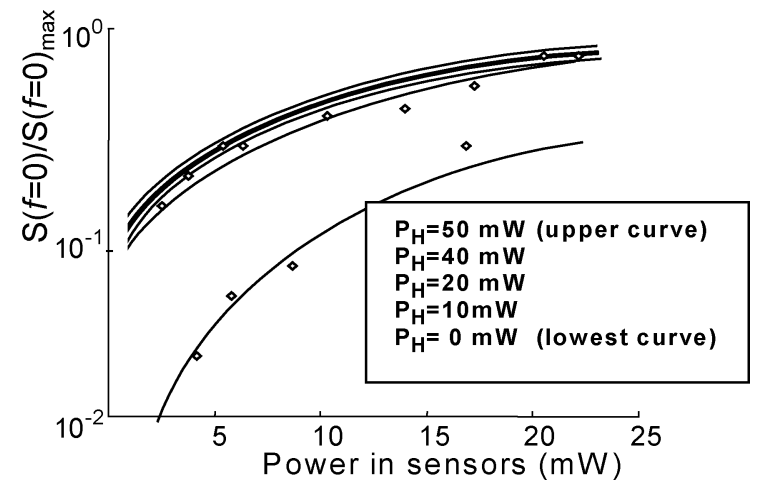

Fig. 9. Relative low-frequency sensitivity $S(f=0)$ as a function of power in the sensors $2 P_{s}$ with the heater power $P_{h}$ as a parameter. The dots represent measurements; the lines model calculations. $P_{h}=0 \mathrm{~mW}$ (lowest curve); 10 ; 20; 30; 40; and $50 \mathrm{~mW}$ (upper curve).

The inverse of the sensitivity to noise ratio, N/S, represents the intrinsic noise or "selfnoise" in $\mathrm{m} /(\mathrm{s} \sqrt{ } \mathrm{Hz})$, with $S$ the sensitivity in $\mathrm{V} /(\mathrm{m} / \mathrm{s})$ and $N$ the noise in $\mathrm{V} / \sqrt{ } \mathrm{Hz}$. It corresponds to an equivalent particle velocity of the noise level. Fig. 10 shows these levels of a three-wire device for varying $r$. The lowest curve shows the minimal selfnoise level at $r \approx 0.85$; the upper curve with $r=0$ represents the two-wire situation.

\section{An Optimally Performing Three-Wire Device}

Comparing Figs. 4, 5, and 6, one sees that the requirement of high low-frequency sensitivity and high characteristic frequency (large bandwidth) may lead to conflicting conditions for the wire separation $a$ : for a large bandwidth $a$ should be as small as possible, but if $a$ is very small, $S_{f=0}$ decreases. The optimization strategy for a large required bandwidth should be to choose a small value of $a$, then determine $l_{z}$, and then $l_{y}$. For an optimum low-frequency $\mathrm{S} / \mathrm{N}$ ratio, $l_{y}$ must be high, thus determining $l_{z}$ and then $a$.

We designed a three-wire device for a high $\mathrm{S} / \mathrm{N}$ and a broad frequency band, with $a=50 \mu \mathrm{m}, l_{z}=200, l_{y}=1000 \mu \mathrm{m}$, $R_{h}=256 \Omega, R_{s 1}=R_{s 2}=3.9 \mathrm{k} \Omega$ at $T=293 \mathrm{~K}$, $P_{h}=58.2 \mathrm{~mW}$, and $P_{s 2}+P_{s 1}=11.5 \mathrm{~mW}$. The temperature of the heater at this power dissipation, reaches approximately $780 \mathrm{~K}$ and the sensor wire temperature is about $327 \mathrm{~K}$. The heater power can be relatively large and it is limited by the maximum temperature the heater can stand. We found

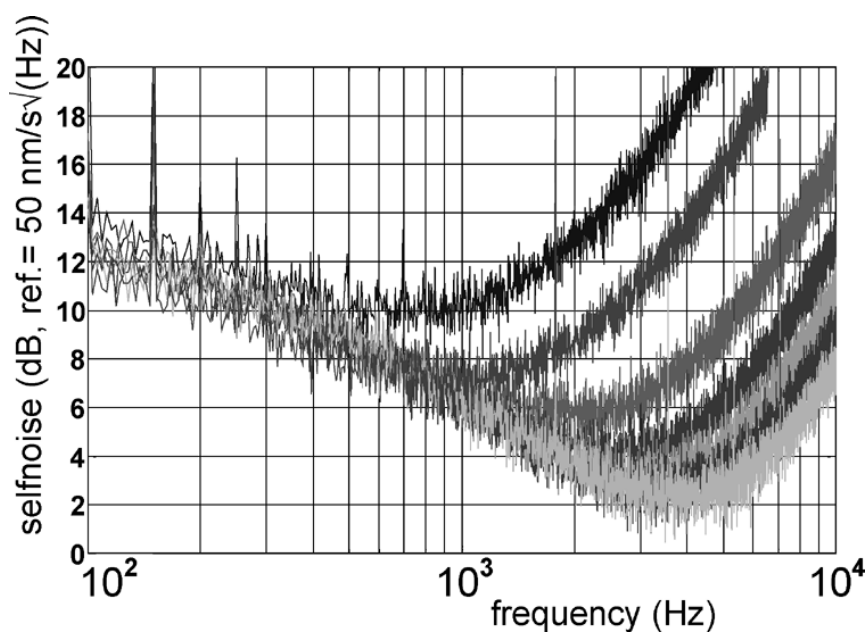

Fig. 10. Selfnoise levels of the three-wire sensor in $\mathrm{dB}$ (the reference level is $50 \mathrm{~nm} / \mathrm{s} \sqrt{ } \mathrm{Hz}$. This corresponds to $0 \mathrm{~dB}$ PVL or SPL, i.e., $20 \mu \mathrm{Pa}$ at a "free field" specific acoustic impedance) the sensor power is $P_{s 1}+P_{s 2}=11.5$, the heater power $P_{h}=0$ (upper curve), $5,10,20,32,44$, and $58 \mathrm{~mW}$ (lowest curve), respectively. $R_{h}=7.90 \mathrm{k} \Omega, R_{s 1}=R_{s 2}=256 \Omega$ at room temperature, $a=100, l_{z}=300 \mu \mathrm{m}$.

$S_{f=0}=0.57 \mathrm{~V} /(\mathrm{m} / \mathrm{s})$, a sensitivity of $0.54 \mathrm{~V} /(\mathrm{m} / \mathrm{s})$ at $1 \mathrm{kHz}$, and an estimated corner frequency of $4.3 \mathrm{kHz}$. As we see in Fig. 10, its selfnoise at $r \approx 0.85$ is $6 \cdot 10^{-8}$ to $2 \cdot 10^{-7} \mathrm{~m} /(\mathrm{s} \sqrt{ } \mathrm{Hz})$ in the frequency bandwidth of 1 to $4 \mathrm{kHz}$, an important audible range.

\section{DISCUSSION AND CONCLUSION}

Starting from principal physical equations, the wire temperature and the response signal due to (acoustic or fluid) flow of the thermal flow sensor have been calculated. Different device geometries have been analyzed numerically. This approach takes into account the finite etch depth, contrary to the previously developed analytic model. The geometric dependences and the power dependence of the sensitivity were experimentally verified, yielding a good correspondence between model and experiment. It is found that for given wire length $l_{y}$, the low frequency sensitivity increases with channel depth $l_{z}$ up to $l_{z} \approx l_{y}$. The optimum wire separation $a$ at a given $l_{z}$ is found to be about $a \approx 0.3 l_{z}$. However, to obtain a broad, flat frequency response, $a$ is chosen to be as small as possible. Both requirements for $a$ cannot be satisfied simultaneously, but a good two-wire device is made if $a \approx 60 \mu \mathrm{m}$ at $l_{y}=500$ and $l_{z}=200 \mu \mathrm{m}$. Next it was seen that for $l_{z}>l_{y}$ the influence of the channel surfaces acting as heat sinks is negligible. The reason for this is that for $l_{z}>l_{y}$ the smaller length $l_{y}$ becomes the characteristic parameter for the geometry and there is no essential difference to a geometry with wires in free space.

Since the $\mathrm{S} / \mathrm{N}$ ratio increases with power, $P$ should be as high as possible. The power per unit length is limited by the temperature limitations of the thin sensor wire, about $650 \mathrm{~K}$ for a platinum wire on silicon nitride.

A significantly better performing device is made of three instead of two wires. The relatively thick central wire, the heater, is heated up to $\sim 780 \mathrm{~K}$, while the other two wires, acting as sensing wires, operate at a relatively low temperature and thus have a lower noise level. With a relative heater power of 0.85 , the sensitivity of a three-wire sensor is approximately two times 
higher sensitivity than a two-wire sensor of the same dimensions and power. We found an optimal geometry with $l_{y}=1 \mathrm{~mm}$, $l_{z}=300 \mu \mathrm{m}$, heater-sensor distance of $50 \mu \mathrm{m}$, and $P_{\text {total }}=$ $70 \mathrm{~mW}$. In the frequency bandwidth of $1 \mathrm{to} 4 \mathrm{kHz}$, the selfnoise of this device is about $6 \cdot 10^{-8}$ to $2 \cdot 10^{-7} \mathrm{~m} /(\mathrm{s} \sqrt{ } \mathrm{Hz})$.

\section{ACKNOWLEDGMENT}

The authors would like to thank M. de Boer, J. W. Berenschot, P. Ekkels, H. Kuipers, and D. R. Yntema for their technical help, practical contributions, and support.

\section{REFERENCES}

[1] H.-E. de Bree, P. J. Leussink, M. T. Korthorst, H. V. Jansen, T. S. J. Lammerink, and M. C. Elwenspoek, "The microflown: a novel device measuring acoustical flows," Sens. Actuators A, vol. 54, pp. 552-557, 1996.

[2] H.-E. de Bree, H.-E. Lammerink, H.-E. Elwenspoek, and H.-E. Fluitman, "Use of a fluid flow measuring device as a microphone and system comprising such a microphone," Patent PCT/NL95/00 220.

[3] W. F. Druyvesteyn et al., "A new sound intensity probe; comparison to the Bruel \& Kjaer p-p probe," J. Audio Eng. Soc., vol. 48, no. 1/2, 2000.

[4] - A New Acoustic Measurement Probe; The Microflown. London, U.K.: IOA, 1999.

[5] H.-E. de Bree et al., "Three-dimensional sound intensity measurements using microflown particle velocity sensors," in MEMS '99, Orlando, FL.

[6] _ - "Realization and calibration of a novel half inch p-u sound intensity probe," in 106th AES Munchen, 1999.

[7] H. Schurer et al., "Comparison of two methods for measurement of horn input impedance," in Proc. 100th AES Convention, Copenhagen, Denmark, 1996.

[8] F. J. M. van der Eerden et al., "Experiments with a new acoustic particle velocity sensor in an impedance tube," Sens. Actuators, vol. A69, pp. 126-133, 1998

[9] H.-E. de Bree et al., A method to measure apparent acoustic pressure, flow gradient and acoustic intensity using two micromachined flow microphones, in Eurosensors X, Leuven, Sweden, 1996.

[10] H.-E. de Bree, "Add-on microflown for a high-end pressure gradient microphone," in Proc. 109th AES Convention, Los Angeles, CA, 2000.

[11] H.-E. de Bree et al., "Bi-directional fast flow sensor with a large dynamic range," JMM, pp. 186-189, 1999.

[12] C. Yang and H. Søeberg, "Monolithic flow sensor for measuring milliliter per minute flow," Sens. Actuators A, vol. 33, pp. 143-153, 1992.

[13] K. Petersen et al., "High precision, high performance mass-flow sensor with integrated laminar flow micro-channels," Sens. Actuators, pp. 361-363, 1985.

[14] G. B. Hocker, "A microtransducer for air flow and differential pressure sensing applications," Micromach. Micropack. Transducers, pp. 207-214, 1985.

[15] R. G. Johnson et al., "A highly sensitive silicon chip microtransducer for air flow and differential pressure applications," Sens. Actuators, vol. 11, pp. 63-72, 1987.

[16] V. B. Svetovoy and I. A. Winter, "Model of the $\mu$-flown microphone," Sens. Actuators, vol. 86, pp. 171-181, 2001.

[17] J. W. van Honschoten, G. J. M. Krijnen, V. B. Svetovoy, H.-E. de Bree, and M. C. Elwenspoek, "Optimization of a two-wire thermal sensor for flow and sound measurements," in Proc. MEMS, Switzerland, 2001.

[18] J. W. van Honschoten, G. J. M. Krijnen, V. B. Svetovoy, H.-E. de Bree, and M. Elwenspoek, "Analytic model of a two-wire thermal sensor for flow and sound measurements," J. Micromechanics, 2004.

[19] A. D. Pierce, Acoustics: An Introduction to Its Physical Principles and Applications. Woodbury, NY: Acoust. Soc. America, 1989.

[20] M. S. Howe, Acoustics of Fluid-Structure Interactions. Cambridge, U.K.: Cambridge University Press, 1998.

[21] R. K. Pathria, Statistical Mechanics, Canada: Univ. Waterloo, 1986.

[22] A. van der Ziel, Noise, Prentice-Hall Electrical Engineering Series, The Netherlands.

[23] P. Dutta and P. M. Horn, "Low-frequency fluctuations in solids: $1 / f$ noise," Rev. Modern Phys., vol. 53, no. 3, 1981.

[24] G. Grinstein, T. Hwa, and H. J. Jensen, " $1 / f^{a}$ noise in dissipative transport," Phys. Rev. A, vol. 45, no. 2, 1992.

[25] S. H. Liu, "Theory of $1 / f$ noise in metal films and whiskers," Phys. Rev. $B$, vol. 16 , no. 10,1977 .
[26] J. H. Scofield, J. V. Mantese, and W. W. Webb, " $1 / f$ noise of metals: A case for extrinsic origin," Phys. Rev. B, vol. 32, no. 2, 1985

[27] E. Milotti, "Linear processes that produce $1 / f$ or flicker noise," Phys. Rev. $E$, vol. 51 , no. $4,1995$.

[28] M. B. Weissman, " $1 / f$ noise and other slow, nonexponential kinetics in condensed matter," Rev. Modern Phys., vol. 60, no. 2, 1988.

[29] J. P. Holman, Heat Transfer, 9th ed: McGregor and Emery, 2002.

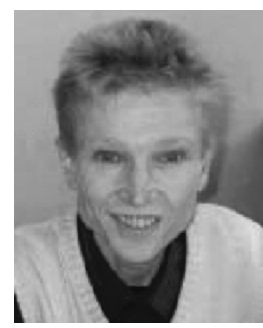

Joost W. van Honschoten was born in Deventer, The Netherlands, in 1972. He studied applied physics at the University of Twente, Enschede, The Netherlands, where he received the M.Sc. degree in 1997, during which period he worked on solitary waves in optical guides and on coherence effects in laser Doppler blood perfusion measurements. $\mathrm{He}$ received the Ph.D. degree in 2004.

His Ph.D. degree research involved the modeling and optimization of an acoustic hot-wire flow sensor, on which he is also working currently.

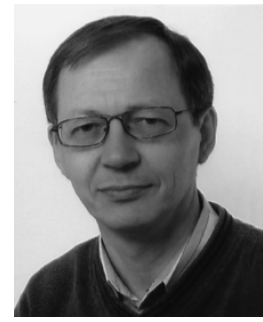

Vitaly B. Svetovoy was born in Yaroslavl, Russia, in 1955. He received the M.S. degree in theoretical and mathematical physics from Yaroslavl University in 1978, and the Ph.D. degree in high energy physics from the Belorussian University, Minsk, in 1985.

$\mathrm{He}$ joined the Institute of Microelectronics in 1985 and was head of the research group since 1989 During the past decade, he was active in silicon etching by plasma, laser direct writing in polymers, and properties of porous silicon. His current research interests are in measurements of very weak forces with the help of microtechhnologies.

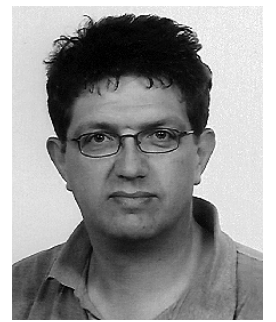

Gijs J. M. Krijnen received the M.Sc. degree in electrical engineering from the University of Twente, Enschede, The Netherlands, following a study on magnetic recording carried out at the Philips Research Laboratories, Eindhoven. For the Ph.D. degree, he worked on nonlinear integrated optics devices.

From 1992 to 1995, he was a Fellow of the Royal Netherlands Academy of Arts and Sciences and studied second- and third-order nonlinear integrated optics devices. In this period, he was a visiting scientist at the Center for Research and Education in Optics and Lasers, Orlando, FL. During 1995-1997, he worked on integrated optic devices for optical telecommunication simultaneously at the University of Twente and the Delft University of Technology. Since 1998, he has been a staff member of the Micro-Mechanics Group of the MESA+ Research Institute. His current interests include (modeling of) MEMS and MOEMS devices in general and microactuators in particular.

Dr. Krijnen was the 1993 recipient of the Veder price of the Dutch Electronics and Radio Engineering Society (NERG).

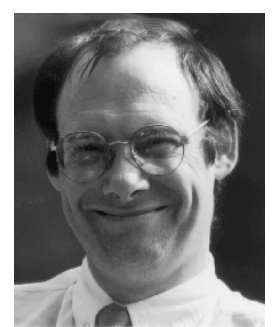

Miko C. Elwenspoek was born in Eutin, Germany, in 1948. He studied physics at the Free University of Berlin, Berlin, Germany, and received the Ph.D. degree from the University of Berlin in 1983. His Ph.D. degree research involved relaxation measurements on liquid metals and alloys, in particular, alkali metal alloys.

From 1977 to 1979 , he was involved with the study of lipid double layers. In 1983, he began studying crystal growth of organic crystals at the University of Nijmegen, The Netherlands. In 1987, he joined the University of Twente, Enschede, The Netherlands, to take charge of the Tansducers and Science Technology Group, MESA + Research Institute. He has been a full professor since 1996. His research interests include fabrication techniques such as the physical chemistry of wet anisotropical etching, reactive ion etching, wafer bonding, chemical-mechanical polishing, and the materials science of various thin films. 